\title{
Application of Transition Prediction
}

\author{
A. KRUMBEIN \\ Deutsches Zentrum für Luft- und Raumfahrt e.V., Institut für Aerodynamik und Strömungstechnik, Numerische \\ Verfahren, Bunsenstraße 10, D-37073 Göttingen, Germany, andreas.krumbein@dlr.de
}

\begin{abstract}
Summary
A Reynolds-averaged Navier-Stokes solver, a laminar boundary-layer code and different transition prediction methods for the prediction of Tollmien-Schlichting and cross flow instabilities were coupled for the automatic prediction of laminar-turbulent transition on general 3-dimensional aircraft configurations during the ongoing flow computation. In this article, the procedure is applied to a two-dimensional three-element high-lift airfoil configuration which is characterized by the existence of laminar separation bubbles. The automatic transition prediction procedure is applied using different operation modes and different transition prediction strategies.
\end{abstract}

\section{Introduction}

The modelling of laminar-turbulent transition in Reynolds-averaged Navier-Stokes (RANS) solvers is a crucial issue when high quality simulation results for aircraft shall be produced. Especially the simulation of flows around high-lift systems of aircraft may result in significant errors when the transition points are of insufficient accuracy or are not taken into account at all. High-lift systems very often involve multi-component wings (e.g. slat, main wing, and flaps) and may have very high levels of total circulation. Because all components of the high-lift system are in close interaction with one another the total circulation and the complete flow field is affected by the transition line on any of the components.

Although the overall lift value may be predicted with satisfactory accuracy slight deviations between the real and the computed pressures can lead to large errors in the computed overall drag value. It could be shown that the overall pressure drag of a high-lift configuration, which dominates the drag value of the configuration as a whole as well as the drag of every single element, is composed of a balance of very large positive and negative contributions, such as the suction forces at the noses or the resistance forces in the coves and the trailing edge regions. The contribution of one single element may be one order of magnitude larger than the resulting overall drag of the complete configuration. Thus, a relative error of $5 \%$ of the computed drag on the slat upper side may result in a change of $50 \%$ for the overall drag value [1].

Another aspect of taking into account transition is that in many cases the high potential of higher order turbulence models can be made use of only when the areas of laminar-turbulent transition are known and deployed in the computational procedures with sufficiently high accuracy. Thus, in modern computational fluid dynamics (CFD) tools a robust transition modelling must be established together with reliable and effective turbulence models. Only if the transition locations are taken into account with sufficient accuracy all physical characteristics of the flow field can be reproduced in such a way that the demanding quality requirements are satisfied.

For the design process of wings, there exists the demand for a RANS-based CFD tool that is able to handle flows automatically and autonomously with laminar-turbulent transition. Existing transition prediction methods vary from empirical transition criteria via the local, linear stability equations based on small disturbance theory or non-local, linear and non-local, non-linear stability methods using the parabolized stability equations over large eddy simulations to direct numerical simulations of the Navier-Stokes equations. Empirical transition criteria and the $\mathrm{e}^{N}-$ method [2],[3] based on local, linear stability theory and the parallel flow assumption represent state-of-the-art methods for the prediction of transition onset in many industrial applications. Although they do not account for a number of fundamental aspects in the transition process $\mathrm{e}^{N}$-methods are used in aircraft industry most frequently for design purposes covering transition due to Tollmien-Schlichting (TS) and cross flow (CF) instabilities. Because there are no other practical methods presently available for industrial applications $\mathrm{e}^{N}$-methods together with the two$\mathrm{N}$ factor method and empirical criteria for transition mechanisms which are not covered by the $\mathrm{e}^{N}$ approach (e.g. bypass and attachment line transition) are going to be used further on for the design of aircraft wings and wing systems even for a future laminar wing of transport type aircraft.

Recently the unstructured/hybrid RANS solver TAU [4] of the Deutsches Zentrum für Luft- und Raumfahrt, German Aerospace Center (DLR) has been provided with a general transition prediction functionality which can be applied to general 3-dimensional aircraft configurations. The developments and first technical validation steps have been carried out at the Institute of Fluid Mechanics (ISM) of the Technical University of Braunschweig (TU-BS), [5],[6]. The TAU code is used together with the laminar boundary-layer method in [7] and the local linear stability code in [8]. These two codes and an infrastructure part of the TAU code are components of a so called 'transition prediction module' that is coupled to the RANS solver and that interacts with the RANS solver during the computation in a very similar way as it is documented in [9].

For a long time it was necessary to use transition database methods in order to apply the $\mathrm{e}^{N}$-method for transition prediction in a fully automatic way so that the transition location iteration could be executed without intervention (automatic) by the user of the RANS code and without a priori knowledge of the transition characteristics of the 
specific flow problem (autonomous). Now the fully automated local, linear stability solver in [8] is available using a frequency estimator for the detection of the relevant regions of amplified disturbances for TS instabilities and a wave length estimator for CF instabilities.

In this article, the coupling structure between the TAU code and the transition prediction module is outlined and the transition prediction strategy is described together with the different operation modes of the transition prediction module which can be selected by the user. The main objective is to demonstrate the different characteristics of the different operation modes and the different transition prediction strategies and their impact on the computational results which are obtained for a two-dimensional three-element high-lift airfoil configuration which is characterized by the existence of laminar separation bubbles for the flow case presented. The computational results are compared to experimental findings.

\section{Transition Prediction Coupling}

On the one hand, the transition prediction module consists of an infrastructure part inside the flow solver which performs pre-processing operations necessary for each step of the transition prediction procedure, for example, the extraction of the surface pressure distribution from a wing section. On the other hand, the module contains a number of additional components which basically execute the transition prediction. These additional components are a laminar boundary-layer (BL) code for swept, tapered wings [7], two $\mathrm{e}^{N}$-database methods, one for TS and the other for CF instabilities [10],[11] and a local, linear stability code [8].

With respect to the calculation of the laminar BL parameters the coupled system can be run in two different modes: Either the TAU code communicates the surface pressure distribution of the configuration to the laminar BL method, the laminar BL method then computes all of the laminar BL parameters that are needed for a selected transition prediction method and the transition prediction method determines new transition locations that are given back to the RANS solver ( $B L$ mode 1$)$. Or the TAU code computes the necessary BL parameters internally and communicates them directly to the transition prediction method (BL mode 2).

Also with respect to the transition prediction $(P D)$ method, the system can be run in two different modes: Either the two $\mathrm{e}^{N}$-database methods ( $P D$ mode 1 ) or the local, linear stability code ( $P D$ mode 2$)$ can be used for the determination of transition points due to TS or CF waves.

This coupled structure results in an iteration procedure for the transition locations within the iterations of the RANS equations. The structure of the approaches using the two different BL modes is outlined graphically in Fig. 1.

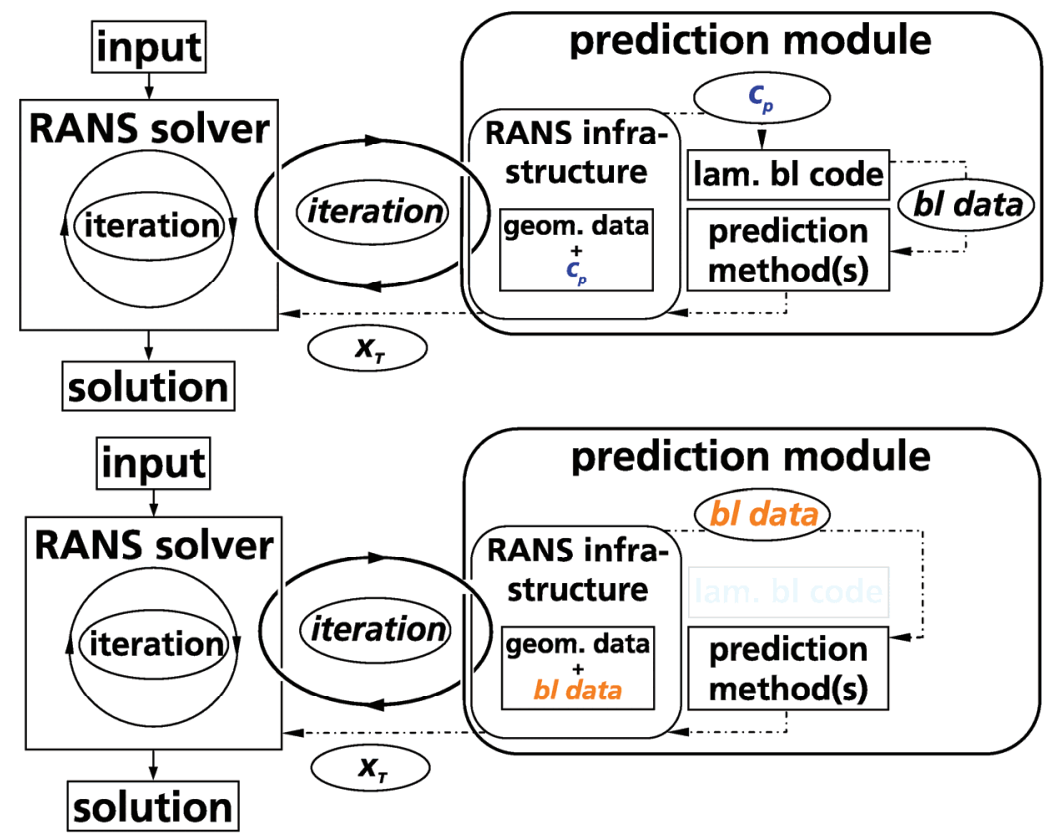

Fig. 1 Coupling structure: $B L$ mode 1 (above) and $B L$ mode 2 (below)

During the computation, the RANS solver is stopped after a certain number of iteration cycles usually when the lift has sufficiently converged, that is when pressure oscillations have been damped to a sufficiently low degree. Then the transition module is called, geometrical data are processed and all laminar viscous data - basically the velocity profiles in streamwise and crossflow direction and their $1^{\text {st }}$ and $2^{\text {nd }}$ derivatives - are calculated either by the $\mathrm{BL}$ code or by the TAU code itself. Then, either the two $\mathrm{e}^{N}$-database methods or the stability code analyze the laminar boundary layer and try to determine a transition point. For BL mode 1 this is possible only when the transition point is located upstream of the separation point predicted by the BL code because the BL code terminates 
when a separation is detected. If a transition point due to TS or CF instabilities was found it is communicated back to the RANS solver. If no transition point due to TS or CF instabilities upstream of the laminar separation point could be found the laminar separation point is used as approximation of the real transition point. This is an attempt to predict transition due strictly to the presence of separation bubbles. This approach often yields a good approximation of the real transition point when transition does not occur before the laminar boundary layer separates, particularly for low Reynolds number flows. For BL mode 2 the laminar BL data are calculated beyond the point of laminar separation which is detected by the TAU code inside the RANS computational grid. Thus, transition inside laminar separation bubbles can be detected without relying on an approximation. Practically, the determination of transition inside laminar separation bubbles is only realizable using $P D$ mode 2 because the $\mathrm{e}^{N}$ database methods lack parameters for the base flow profiles in laminar separation bubbles.

These steps are done for the upper and lower sides of all specified wing sections. When all new transition locations have been communicated back to the RANS solver, each transition location is slightly underrelaxed to damp oscillations in the convergence history of the transition locations. Then, all underrelaxed transition points they represent a transition line on the upper or lower surface of a wing element in form of a polygonial line - are mapped onto the surface grid of the configuration applying a transition setting algorithm subdividing the surface of the geometry into laminar and turbulent regions, and the computation is continued. In so doing, the determination of the transition locations becomes an iteration process itself. With each transition location iteration step the underrelaxation factor is reduced until a converged state of all transition points has been obtained.

\section{Computational Results}

The system was applied to the A310 take-off configuration [12] consisting of slat, main airfoil and flap defined by $\mathrm{M}=0.221, \mathrm{Re}=6.11 \times 10^{6}$ and $\alpha=21.40^{\circ}$. According to [13], as value for the limiting $\mathrm{N}$ factor for TS instabilities $\mathrm{N}_{\mathrm{T}}=9$ was used. In the experiments [12] the following transition locations were determined on the upper sides of the slat, $\left(\mathrm{x}_{\text {upp }}^{\mathrm{T}} / \mathrm{c}\right)_{\text {slat }}=0.15$, and the flap, $\left(\mathrm{x}_{\text {upp }}^{\mathrm{T}} / \mathrm{c}\right)_{\text {flap }}=0.345$. On the main airfoil upper side the transition location was not measured, but the location of the upper side kink - the point where the slat trailing edge is located when the configuration is undeflected - is useful as a point of orientation, $\left(\mathrm{x}_{\text {kink }}{ }_{\text {upp }} / \mathrm{c}_{\text {main }}=0.19\right.$. On the lower sides, the transition points were not measured. In the computations a standard one-equation turbulence model was applied. The computations were started with free stream initialization and were carried out for two different computational grids [14] exhibiting different grid densities. In the fine grid, the grid resolution was highly increased compared to the coarse grid in streamwise as well as in wall normal direction in the structured parts which resolve the boundary layers. While the coarse grid (grid 1) consists of about 22,000 primary grid points the fine grid (grid 2) has about 122,000 points, Fig. 2.

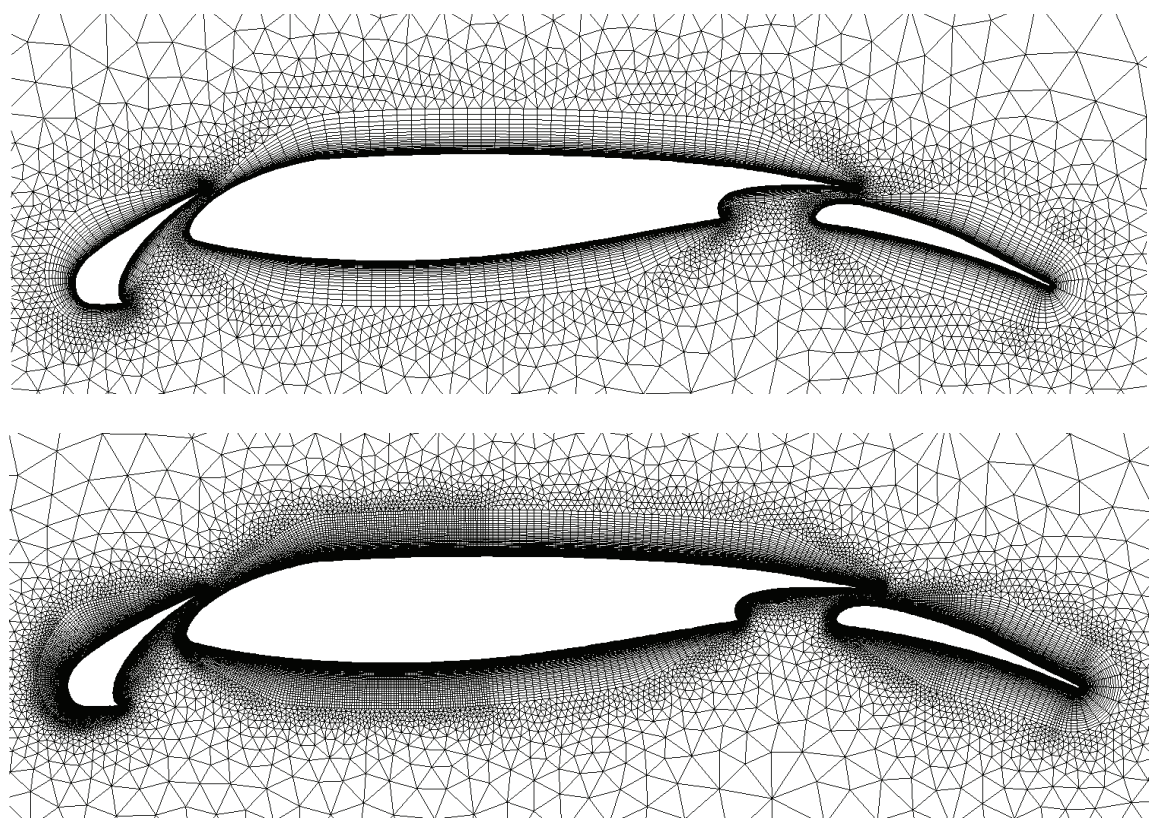

Fig. 2 Computational grids: grid 1 (above), grid 2 (below)

Because in the experiments a laminar separation bubble on the slat upper side caused transition, the computations using grid 2 were intended to resolve the laminar separation bubble and to investigate the impact on the transition locations caused by the different modes of the transition prediction module.

The three different combinations of modes which are currently available in the TAU code for 2-dimensional 
cases are the following: a) BL mode $1 \& P D$ mode 1 , b) BL mode $1 \& P D$ mode 2 and c) BL mode $2 \& P D$ mode 2. For the combinations $\mathrm{b}$ ) and c) an extrapolation of the $\mathrm{N}$ factor curves computed by the linear stability code can be applied. The extrapolation is usually switched off for case b), so that a laminar separation point from the BL code approximates transition when transition due to TS or CF instabilities does not occur. For case c), the extrapolation is usually switched on, because the numerical dissipation in the RANS code affects the quality of the laminar BL data which are calculated inside the RANS grid when case c) is applied. The effect of the numerical dissipation in the RANS code is an upstream influence of the turbulent quantities which start to develop downstream of the current transition point. Nevertheless, they have an influence also on the laminar BL profiles directly upstream of the transition point and give them a slightly bellied shape. Thus, because these influenced BL profiles are interpreted as fully laminar ones by the linear stability code, they are considered as stable or at least stabilized BL profiles. For those surface stations which are influenced in such a way, the values of the $\mathrm{N}$ factor curves tend to decrease, so that it can happen that the critical $\mathrm{N}$ factor is not reached by the envelope of the computed $\mathrm{N}$ factor curves. This disadvantageous effect can the significantly reduced, when the envelope of the $\mathrm{N}$ factor curve is extrapolated from a point where it is not yet affected by this upstream effect. In the TAU code, a linear extrapolation is started from that point on where the steepest slope of the envelope of the $\mathrm{N}$ factor curves is found. This extrapolation is then used to determine the new transition point, which found where the extrapolation curve reaches the critical $\mathrm{N}$ factor.

In this article, results obtained for case b) without extrapolation, for case c) with extrapolation, and for case b) with extrapolation are shown, where the latter combination yields a new prediction strategy that keeps the computational speed of the BL code while getting rid of the approximation of the transition point by a laminar separation point. This new approach can yield much better results when it is favoured or necessary to use the laminar BL code.

The transition prediction procedure which was started with initial transition points located almost at the upper side trailing edges of the particular elements (on the lower sides, fully laminar flow was assumed up to the trailing edges) was run with a pre-prediction phase of 1000 iteration cycles where the laminar separation points which occur in the RANS grid are used as transition points in order to stabilize the computation. The pre-prediction interval was 20 iteration cycles. Then, the transition prediction iteration was started using a prediction interval of 500 iteration cycles.

In Fig. 3, the $c_{p}$ - and $c_{f}$-distributions for grid 1 are shown. As expected, the results from the computations with predicted transition (PD) yield more negative pressure levels on the upper sides of all elements than the fully turbulent (FT) results. This effect is pronounced in the suction peak areas. In terms of the pressure no significant difference between case b) without extrapolation and case b) with extrapolation can be recognised. The comparison of the $c_{f}$-distributions clearly shows the transition from laminar to turbulent flow on all elements. On the main wing element, transition occurs directly upstream of the kink for case b) without extrapolation, on slat and flap upper side the predicted transition points are located clearly upstream of the experimentally determined locations. Especially, on the flap the deviation is remarkable. While there is no effect on the slat when case b) with extrapolation is applied - on the slat, the flow is still very stable, so that no amplified modes can be detected by the linear stability code and the expected laminar separation bubble can not evolve because grid 1 is not fine enough -, significantly different transition location are predicted for the main element and the flap. The transition point on the main element is now located downstream of the upper side kink, the transition location on the flap almost coincides with the experimentally determined transition point. Although this result is based only on a first test case example, it shows that significant improvements of the predicted transition locations can be obtained using the BL code and the extrapolation technique of the envelope of the $\mathrm{N}$ factor curves.

Fig. 4 shows the results for grid 2. For grid 2, the mode combination b) without extrapolation and c) yield converged results. For the computations with transition, a separation bubble on the slat upper side is reproduced. For b) without extrapolation, where the transition point inside the separation bubble was approximated using the laminar separation point from the BL code, the resulting bubble is of too small extent and of too small strength due to the fact that through this kind of approximation the turbulence production starts too far upstream, so that the separation bubble can not fully develop. For mode combination c), where the stability analysis is carried out inside the laminar separation bubble, the extent and strength of the bubble show a good qualitative agreement with the experimental pressures. Whereas for $b$ ) without extrapolation the predicted transition location on the slat upper side is not very different from that in grid 1 , for mode combination c) the measured transition point now is reproduced with excellent accuracy. The transition point on the main wing element is determined downstream of the kink using b) without extrapolation and more upstream of the kink than it was the case for grid 1 when combination c) is used. In the transition region on the main element, the interaction between transition and the influence of the kink lead to a relatively strong change between the $c_{f}$-distributions from mode combination $b$ ) without extrapolation on the one hand and from c) on the other hand. On the flap upper side the transition point resulting from b) without extrapolation shows almost the same deviation from the measured value as for grid 1. 

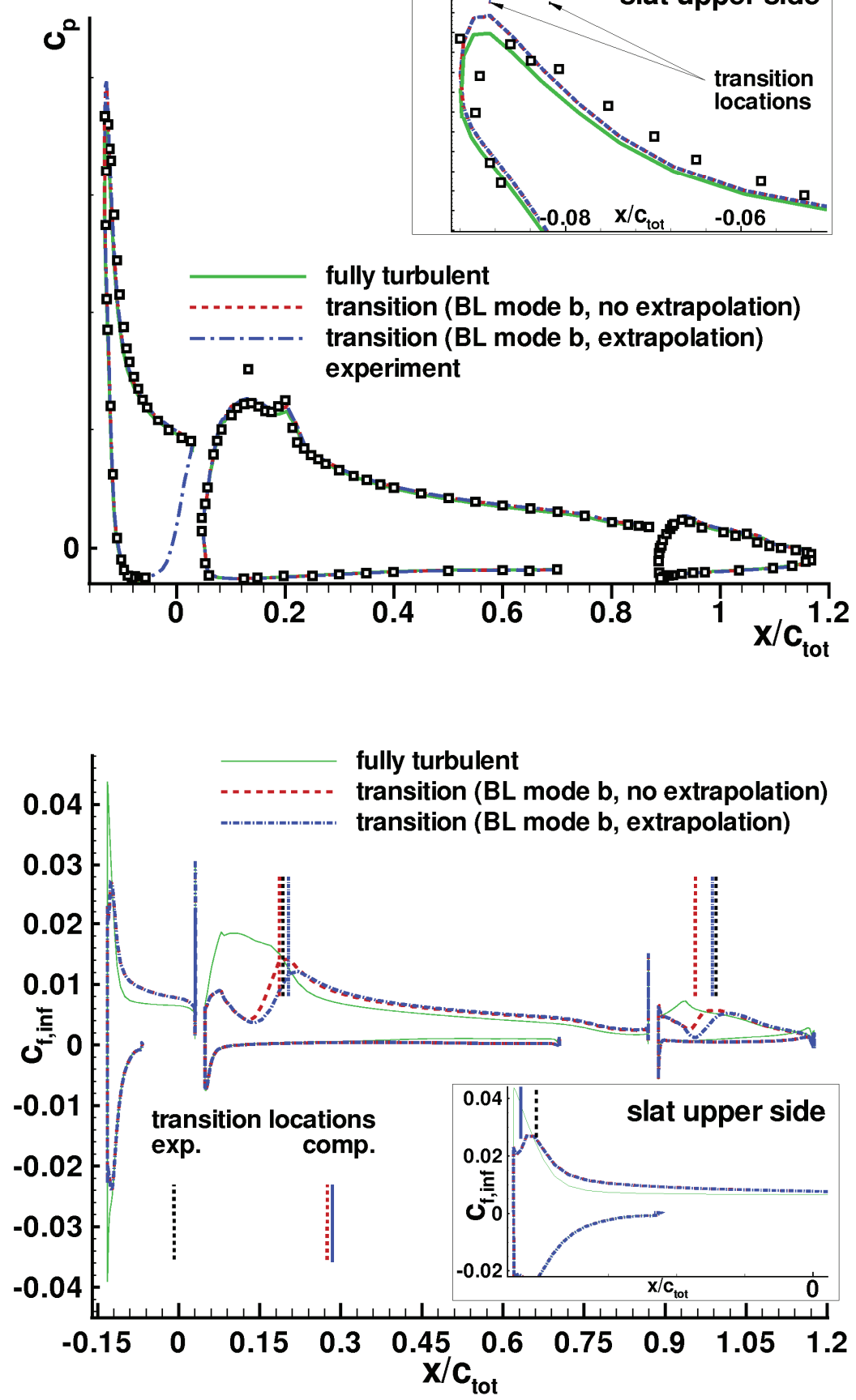

Fig. $3 \mathrm{c}_{\mathrm{p}^{-}}$(above) and $\mathrm{c}_{\mathrm{f}}$-distributions (below) for grid 1

For mode combination c) however a visible downstream shift of the transition point occurs decreasing the gap between the former computational results and the experiment significantly. The error reduction between the predicted and measured flap upper side transition point is about $40 \%$.

In addition to the results using the critical $\mathrm{N}$ factor $\mathrm{N}_{\mathrm{T}}=9$ for the TS instabilities, more results using the value $\mathrm{N}_{\mathrm{T}}=9.8$ were produced for mode combination $\mathrm{c}$ ). The value $\mathrm{N}_{\mathrm{T}}=9.8$ can be found in the literature [15] for the same wind tunnel where the measurements reported in [12] were carried out. As can bee seen in Fig. 4, a further slight improvement of the predicted transition locations on the slat and flap upper sides can be achieved. On the slat, the effect is an increase of the laminar separation bubble in strength and extent. A comparison of the $c_{\mathrm{p}}$-distributions of the results from mode combination c) with $\mathrm{N}_{\mathrm{T}}=9$ and $\mathrm{N}_{\mathrm{T}}=9.8$ shows that only a very small shift of the transition location inside the bubble can have a visible effect on the pressure in that area and, thus, can influence easily the shape and size of the bubble and the strength of the separation in the simulation. 

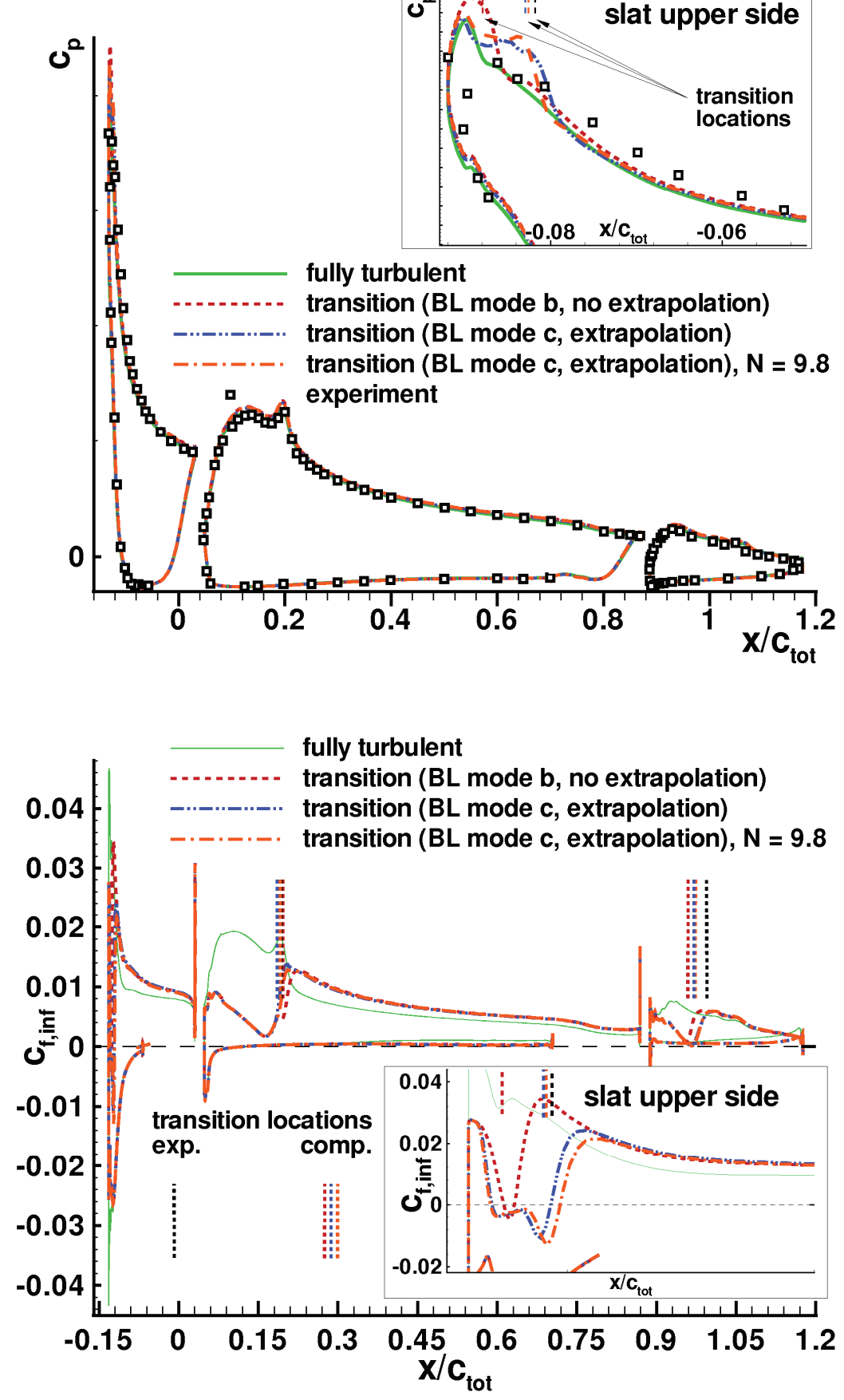

Fig. $4 c_{p^{-}}$(above) and $c_{\mathrm{f}^{-}}$-distributions (below) for grid 2

An inspection of the skin friction distribution in the transition area of the flap upper side of the results obtained with mode combination c) reveals that the boundary layer is shortly before separation as shown in Fig. 5. In the test report [12] it is documented that transition on the slat upper side was provoked by a laminar separation bubble in which the transition occurs. For the flap transition location, there is no indication in the test report that this could be the case too. In the test report a clear distinction is made between transition locations which occur inside a separation bubble and other transition locations for which separation could not be observed. Thus, for the upper side flap it was always assumed that transition occurred due to a TS instability. If this assumption is true the simulation using the prediction approach based on mode combination b) without extrapolation is expected to yield a much better accuracy of the transition location as is obtained in the result presented in this article or in other publications. But this is not the case. The prediction approach which uses a laminar separation point as an approximation of the 
transition point fails and predicts a transition point which is located significantly too far upstream. It is commonly accepted that a laminar boundary layer code is better suited than a RANS code to predict the point of the onset of a laminar separation. This is due to the fact that, on the one hand, the parabolic character of the boundary layer equations prevents any upstream influence, an effect which is always disadvantageous in a RANS simulation with transition when a laminar separation point is located in the direct vicinity of a transition point. On the other hand, the boundary layer codes used for transition prediction purposes use many points in the wall normal direction to resolve the laminar boundary layers - the BL code in the TAU transition prediction module uses 100 points - and apply a fourth-order accurate finite difference scheme in wall normal direction for the discretisation of the BL profiles, in contrast to a nominally second-order accurate discretisation scheme in the RANS codes. These properties of the laminar BL codes and the experience that laminar separation predictions using BL codes very often give clearly better results than those using RANS codes - normally, a laminar separation point predicted by a laminar BL code is located further upstream than that of a RANS code when the same point distribution on the surface is used - have provided much confidence in the correctness of these results. Also the author is not in doubt about that.

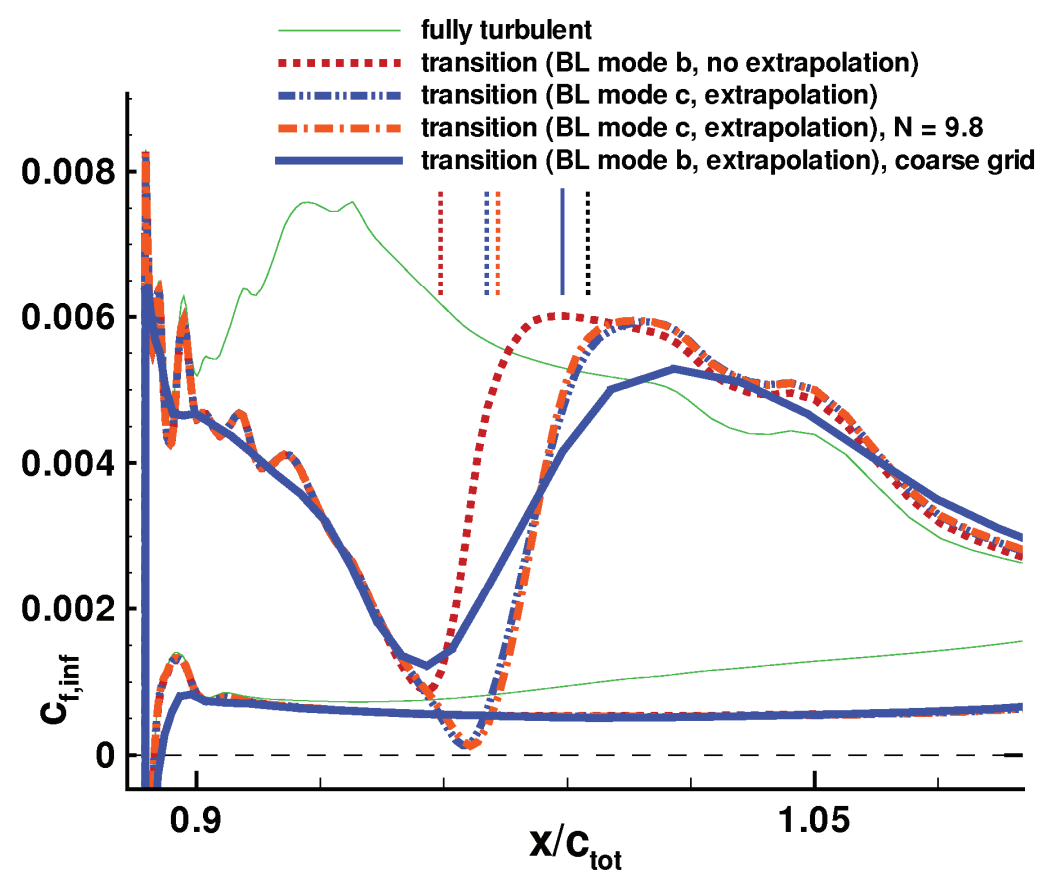

Fig. $5 \mathrm{c}_{\mathrm{f}}$-distributions on the flap for grid 2

Now it turns out that if one does not approximate the transition point by a laminar separation point from a laminar BL code, the results can be significantly improved. In the simulations using mode combination c) no separation occurs on the flap upper side and laminar boundary layer profiles can be computed up to a point where the boundary layer almost separates. Here, again the situation occurs that a laminar BL code predicts laminar separation further upstream than the RANS code does. The upstream influence in the RANS code prevents the separation of the boundary layer and the evolution of a separation bubble. Because the upstream influence is an effect which is highly dependent on the numerical dissipation it is very probable that a further refinement of the grid - in streamwise as well as in wall normal direction - will improve the results once more and will lead to an evolution of a separation bubble. When this happens, the transition module computes laminar boundary-layer profiles beyond the laminar separation point and will detect transition inside the bubble in the same way as it was done for the slat upper side. If this supposition is true must still be verified.

In addition to the results for grid 2 (fine), the results for grid 1 (coarse) from the computation with mode combination b) with extrapolation are shown in Fig. 5. Here, the upstream effect becomes particularly apparent and is accompanied by an error reduction between the predicted and measured flap upper side transition point of about $80 \%$.

In Fig. 6, the velocity profiles in the area of the transition point on the flap for grid 1 and mode combination b) with extrapolation and grid 2 and mode combination c) for $\mathrm{N}_{\mathrm{T}}=9$ and $\mathrm{N}_{\mathrm{T}}=9.8$ are shown. The corresponding transition locations are marked. The result for grid 1 explicitly demonstrates the upstream effect which gives the laminar boundary-layer profiles a slightly bellied shape that they would not have without this effect. 

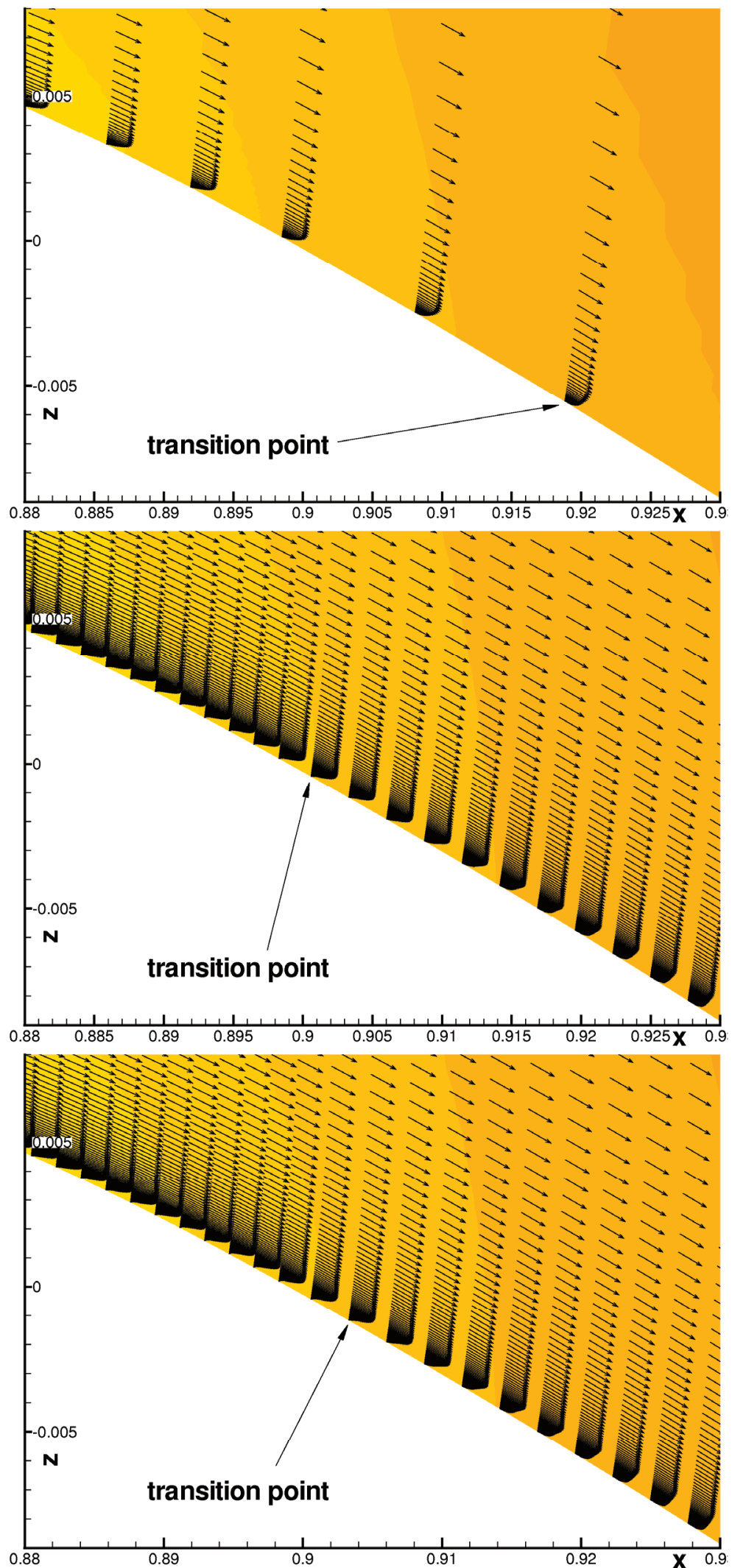

Fig. 6 Velocity profiles in the flap transition region: grid 1 (above), grid 2 and $\mathrm{N}_{\mathrm{T}}=9$ (middle), grid 2 and $\mathrm{N}_{\mathrm{T}}=9.8$ (below), $\mathrm{x}$ - and z-scales are independent

A close inspection of the results for grid 2 shows that about three profiles upstream of the respective transition point are affected by the upstream effect with increasing absolute values of the velocity vectors at the first grid point apart from the wall. 
In addition to the verification that a finer grid resolution leads to the expected laminar separation bubble on the flap upper side two more things have to be done in order to fully conclude this investigation: The first is the application of mode combination b) with extrapolation in a computation using grid 2 (fine). The second is a test of a mixture of the mode combinations where the external (BL code) and the internal (from the RANS grid) computation of the boundary-layer data with and without extrapolation are applied in different combinations to the three different elements of the configuration.

\section{Conclusion}

The TAU code coupled to a newly developed transition prediction module was applied to a two-dimensional three-element high-lift airfoil configuration which is characterized by the existence of laminar separation bubbles. The prediction of the transition location was carried out in a fully automatic manner during the ongoing RANS computation so that no intervention of the user is needed. It could be shown that when the computational grid is fine enough transition locations inside laminar separation bubbles can be predicted with high accuracy while the separation bubble itself can be reproduced well with respect to its extent and strength.

Different operation modes of the transition prediction module were applied in the investigations. It was shown that the approximation of a transition point by a laminar separation point from a boundary-layer code can result in a poor result for the predicted transition location. This shortcoming can be overcome when the extrapolation of the $\mathrm{N}$ factor curves is used. The extrapolation of the $\mathrm{N}$ factor curves can always be used when the laminar boundary layers are computed from the RANS computational grid which requires a grid resolution which is fine enough in streamwise as well as in wall normal direction. In principle, the extrapolation can also be used when the laminar boundary layers are computed by the laminar boundary-layer code. In this case, however, it can happen that up to the point of laminar separation which is detected by the boundary-layer code the perturbation modes are not amplified so that no $\mathrm{N}$ factor curve exists. In such a case, the internal computation of the boundary layers is the only way to solve the problem.

\section{Acknowledgements}

The development work of the transition prediction module within the TAU code was carried out by the Institute of Fluid Mechanics of the Technical University of Braunschweig within the German Research initiative MEGADESIGN.

\section{References}

[1] Rudnik, R., Ronzheimer, A., and Schenk, M., „Berechnung von zwei- und dreidimensionalen Hochauftriebskonfigurationen durch Lösung der Navier-Stokes Gleichungen“, Jahrbuch 1996 - Bd. II der DGLR - JT 96-104, Deutscher Luft- und Raumfahrtkongreß, DGLR-Jahrestagung Sep. 1996, pp. 717-726.

[2] Smith, A.M.O., Gamberoni, N., "Transition, Pressure Gradient and Stability Theory“, Douglas Aircraft Company, Long Beach, Calif. Rep. ES 26388, 1956.

[3] van Ingen, J.L., "A suggested Semi-Empirical Method for the Calculation of the Boundary Layer Transition Region", University of Delft, Dept. of Aerospace Engineering, Delft, The Netherlands, Rep. VTH-74, 1956.

[4] Kroll, N., Rossow, C.-C., Schwamborn, D., Becker, K., and Heller, G., "MEGAFLOW - A Numerical Flow Simulation Tool For Transport Aircraft Design", ICAS Congress 2002 [CD-Rom], ICAS, Toronto, Canada, 2002, pp. 1.105.11.105.20.

[5] Nebel, C., Radespiel, R., and Wolf, T., "Transition Prediction for 3D Flows Using a Reynolds-Averaged Navier-Stokes Code and N-Factor Methods", AIAA-2003-3593.

[6] Krimmelbein, N., Radespiel, R., Nebel, C., "Numerical Aspects of Transition Prediction for Three-Dimensional Configurations", AIAA-2005-4764.

[7] "COCO - A Program to compute Velocity and Temperature Profiles for Local and Nonlocal Stability Analysis of Compressible, Conical Boundary Layers with Suction", ZARM Technik Report, November 1998.

[8] Schrauf, G., "LILO 2.1 User's Guide and Tutorial", Bremen, Germany, GSSC Technical Report 6, originally issued Sep. 2004, modified for Version 2.1 July 2006.

[9] Krumbein, A., "Automatic Transition Prediction and Application to Three-Dimensional Wing Configurations", Journal of Aircraft, Vol. 44, No. 1, 2007, pp. 119-133, DOI: 10.2514/2254; also AIAA Paper 2006-914, June 2006.

[10] Stock, H. W., Degenhardt, E., "A simplified $\mathrm{e}^{N}$ method for transition prediction in two-dimensional, incompressible boundary layers“, Zeitung für Flugwissenschaft und Weltraumforschung, Vol. 13, 1989, pp. 16-30.

[11] Casalis, G., Arnal, D., "ELFIN II Subtask 2.3: Database method - Development and validation of the simplified method for pure crossflow instability at low speed", ELFIN II - European Laminar Flow Investigation, Technical Report $n^{\circ} 145$, ONERA-CERT, Département d'Études et de Recherches en Aérothermodynamique (DERAT), R.T. DERAT $\mathrm{n}^{\circ}$ 119/5618.16, December 1996

[12] Manie, F., Piccin, O., Ray, J.P., "Test Report of the 2D Model M1 in the ONERA F1 Wind Tunnel”, GARTEUR AD(AG08), TP-041, 1989.

[13] Arthur, M. T., Dol, H., Krumbein, A., Houdeville, R., Ponsin, J., "Application of Transition Criteria in Navier-Stokes Computations", GARTEUR AD(AG-35), TP-137, 2003.

[14] Wild, J., private communications, DLR, Institute of Aerodynamics and Flow Technology, Braunschweig, Germany, Nov. 2003 \& Nov. 2005

[15] Cliquet, J., Houdeville, R., Arnal, D., "Application of Laminar-Turbulent Transition Criteria in Navier-Stokes Computation", AIAA-2007-515 